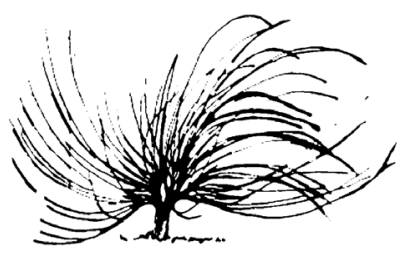

\title{
La pedagogía crítica de Paulo Freire y sus aportes a la educación superior costarricense actual
}

\author{
Manuel Gerardo Espinoza Arroyo ${ }^{l}$ \\ Universidad Nacional \\ Costa Rica \\ manesp94@gmail.com
}

\begin{abstract}
Resumen
En la actualidad, la educación superior en Costa Rica enfrenta una serie de desafíos que atentan contra su autonomía e institucionalidad en general. En este contexto, el presente escrito tiene como objetivo principal analizar la propuesta pedagógica de Paulo Freire y sus aportes a la educación superior costarricense. En este ensayo, se profundizará en tres aspectos que el pedagogo brasileño aporta a la universidad pública costarricense: el diálogo, el pensamiento dialéctico y la transformación. Cada uno de estos se explicará desde la postura freireana, a la vez que se reflexiona sobre la aplicación de cada uno de ellos en el contexto costarricense.
\end{abstract}

Palabras clave: Costa Rica, educación superior, Paulo Freire, pedagogía crítica

\begin{abstract}
Costa Rican higher education at present faces a series of challenges that attempt against its general autonomy and institutionality. Therefore, in this context, this paper has
\end{abstract}

Recibido: 19 de agosto de 2020. Aprobado: 1 de junio de 2021

http://dx.doi.org/10.15359/rep.16-2.5

1 Estudiante Asistente del Instituto de Estudios Latinoamericanos de la Universidad Nacional. Bachiller en la Enseñanza de los Estudios Sociales y Educación Cívica de la Universidad Nacional. Licenciado en Ciencias Religiosas por la Universidad Da Vinci de Guatemala. Actualmente, cursa la Maestría en Educación con énfasis en Pedagogía Universitaria en la Universidad Nacional. ORCID: https://orcid.org/0000-0002-8437-3052 
the main objective to analyze Paulo Freire's pedagogical proposal and his educational contributions to Costa Rican higher education nowadays. Consequently, this essay seeks to deepen three aspects, which the Brazilian pedagogue contributes to public Costa Rican universities: dialogue, dialectical thought, and transformation. In addition, every single aspect will be explained from the Freirean viewpoint in addition to thinking about the enforcement of it in the Costa Rican context.

Keywords: Costa Rica, critical pedagogy, higher education, Paulo Freire

En verdad, lo que pretenden los opresores es transformar la mentalidad de los oprimidos y no la situación que los oprime. A fin de lograr una mejor adaptación a la situación que, a la vez, permita una mejor forma de dominación. (Freire, 2005, p. 81)

\section{Introducción}

$\mathrm{E}$ 1 presente ensayo surgió a partir de un proceso de análisis y reflexión que se llevó a cabo durante el módulo Fundamentos Teóricos y Epistemológicos de la Pedagogía Universitaria, de la Maestría en Educación con énfasis en Pedagogía Universitaria de la Universidad Nacional de Costa Rica. Durante este proceso de enseñanza y aprendizaje, se realizaron diferentes actividades, las cuales favorecieron la construcción de esta publicación. Este estudio tiene como objetivo principal analizar la propuesta pedagógica de Paulo Freire y sus aportes a la educación superior costarricense en la actualidad.

En este sentido, la pedagogía crítica de Paulo Freire ha nutrido el pensamiento pedagógico, no solamente latinoamericano, sino también en diferentes partes del mundo. Por tal motivo, como uno de los primeros exponentes de las llamadas pedagogías críticas, es indispensable conocer sus propuestas, aportes y pensamientos en torno a la tarea de la enseñanza. Sobre todo, el conocimiento de su propuesta no debe ser únicamente para acumular información, más bien debe ser llevado a la práctica. Por consiguiente, la educación superior costarricense puede y debe ser un espacio pertinente para tratar de ejercer las ideas del pedagogo brasileño. 
De esta manera, en este trabajo se pretende abordar la realidad de la educación superior costarricense, la cual tiene un papel de suma importancia en la formación de las y los profesionales nacionales. Sin embargo, en la actualidad se evidencia una fuerte persecución contra la institucionalidad universitaria, lo cual pone en peligro la autonomía con la que esta cuenta y el presupuesto que se le asigna anualmente. Por esta razón, es necesario y urgente escribir acerca de la universidad y cómo, desde la pedagogía crítica de Freire, puede ser fortalecida en las aulas.

De acuerdo con lo anterior, se presenta en este documento una explicación sobre la propuesta pedagógica de Paulo Freire, desde sus propios escritos, en especial su libro Pedagogía del Oprimido, y la interpretación que algunos autores han realizado sobre el pensamiento pedagógico freiriano. Todo esto favorecerá el análisis de los aspectos que esta pedagogía crítica puede aportar a la educación costarricense actual, lo cual busca, desde luego, la transformación de los integrantes de la comunidad educativa, y con ello, el cambio indispensable en las esferas sociales.

\section{Realidad actual en Costa Rica}

En Costa Rica, desde la década de los ochenta, a partir de la implementación de los Programas de Ajuste Estructural, el neoliberalismo se convirtió en la visión hegemónica en la formulación y ejecución de políticas públicas. Por tanto, en la actualidad, el poder político, más allá de intentar establecer la visión neoliberal, lo que pretende es asegurar su vigencia, y para esto utiliza todos los mecanismos posibles, lo que incluye a los espacios educativos de primaria, secundaria y educación superior. En este contexto, cobra real importancia el conocimiento y la práctica sobre la propuesta pedagógica de Paulo Freire, la cual busca la liberación de las masas oprimidas y la transformación de la sociedad.

Las pedagogías críticas denuncian el reproduccionismo que las clases dominantes realizan por medio de la enseñanza. Esto se logra con el control del currículo y de la producción y transmisión que se hace a través de los libros de texto (Carbonell, 2015). Es claro que los que gobiernan lo político y económico establecen formas para legitimar sus diversos proyectos, a favor de sí mismos, dejando de lado a la mayoría de la población. Estos grupos se sirven de la educación como medio para justificar sus proyectos socioeconómicos. Por esta razón, 
las pedagogías críticas buscan convertir la escuela en una esfera pública democrática, en donde la tarea más importante sea "educar a los estudiantes en el lenguaje de la crítica, la posibilidad y la democracia" (Carbonell, 2015, p. 71).

Lamentablemente, este tipo de educación se ve bajo riesgo por las políticas públicas que se intentan crear y establecer, las cuales, en ocasiones, atentan contra la justicia y el respeto hacia los grupos excluidos. De esta manera, Torres (2017) afirma que "una educación para una sociedad democrática, justa e inclusiva no es posible sin políticas públicas que traten de compensar y corregir todas aquellas injusticias estructurales que inciden y determinan las condiciones de vida de chicos, chicas y familias" (p. 31). Deben ser propuestas que favorezcan la inclusión de todas las personas en las diferentes áreas sociales, y que esta permita la transformación de las personas, y con ello, el cambio social.

No obstante, en las últimas décadas, los planteamientos determinados desde los poderes políticos buscan el desmantelamiento de políticas públicas que benefician a la población vulnerable y excluida. Dentro de este proceso de destrucción del llamado Estado de bienestar y la imposición del sistema capitalista, el cual rige el mercado mundial, se ha intentado afectar, de forma directa, la institucionalidad de la universidad pública costarricense. Actualmente, desde la Asamblea Legislativa se ha procurado establecer proyectos que pretenden reformar la Constitución Política, lo cual afecta el presupuesto y el funcionamiento de las universidades públicas del país (Córdoba, 2020).

La realidad de Costa Rica en la actualidad es la de un país que busca sin cansancio la implementación brutal y violenta del modelo económico neoliberal. Para esto, es indispensable controlar la universidad pública, pues de alguna u otra forma, es concebida como un espacio para la crítica y constante cuestionamiento sobre los poderes estatales (Rivas, 2018). Ante esta realidad, la manipulación de las casas de estudio favorece el control ideológico de las masas, haciendo de las personas, individuos dóciles que no cuestionan ni critican las decisiones impuestas desde arriba (Olivares, 2020).

Además, desde la lógica del neoliberalismo, la educación es concebida a partir del pensamiento del mercado, en donde se realiza toda una inversión en las personas, que con el tiempo se recuperará en el mediano o largo plazo, cuando el individuo ofrezca su servicio al 
sistema económico (Zúñiga, 2011). En este sentido, como lo expone Rivas (2018), esta clase de educación

Se basa en el principio positivista de que todo tiene que ser cuantificado, racionalizado, descrito o planificado y en que además la subjetividad debe jugar un papel de mera expectación, dedicándose a acoger a la "objetividad" sin ningún prejuicio. He aquí la base sobre la cual se asienta la anulación del sujeto. (p. 73)

De acuerdo con lo anterior, se puede afirmar que esta clase de educación universitaria trae consigo grandes problemas para la sociedad, pues como se ha explicado, busca la formación de personas sin pensamiento, criterio u opinión. Simplemente, se plantea que los sujetos respondan de manera positiva a lo que es impuesto desde los que gobiernan. El excesivo control de lo social, cultural y lo político, por parte de los que mantienen el poder (Santos, 2009), afecta de forma directa la vida, dignidad y los derechos de la población. Ante esto, es indispensable conocer un tipo de pedagogía que apoye la formación de sujetos críticos, dispuestos a liberarse de los sistemas opresores, y con ello transformar la realidad social inmediata.

\section{Propuesta pedagógica de Paulo Freire}

Las diversas luchas sociales y políticas que se desarrollaron en la segunda mitad del siglo pasado provocaron el nacimiento, desarrollo y establecimiento de movimientos sociales, los cuales buscaban la constante lucha contra los principios ideológicos del sistema capitalista. Este sistema ganaba mayor terreno en las diferentes naciones del mundo, por lo cual debía resistirse de alguna u otra forma. El capitalismo utiliza los diferentes medios de transmisión de la cultura para reproducir sus principales postulados. De acuerdo con lo anterior, usa los espacios de enseñanza y aprendizaje para mantener el control de la estructura económica de la sociedad (Carbonell, 2015).

Ante todas estas ideas y formas de enseñanza deterministas, surgen las pedagogías críticas, las cuales buscan atribuir "un especial protagonismo al sujeto como agente de cambio y transformación social" (Carbonell, 2015, p. 65). Las pedagogías críticas entienden que todos los sujetos, desde sus diferentes contextos y formas de vida, pueden y 
deben aportar grandes transformaciones para el beneficio de las sociedades. En este sentido, se ha considerado que este tipo de pedagogías plantean ideas utópicas y llenas de grandes sueños, ya que, en muchos casos, los sistemas dominantes alejan a los integrantes del sistema educativo de una participación social realmente activa.

Dentro de los principales exponentes de las pedagogías críticas, se puede mencionar a Paulo Freire, quien además es considerado como uno de los referentes iniciales de estas propuestas pedagógicas (Walsh, 2017). En este sentido,

Freire recupera el planteamiento de Marx, que supera la concepción tanto del idealismo como del materialismo vulgar, de la realidad. Del idealismo supera la primacía del sujeto -de corte solipsista- y del materialismo vulgar que borra por completo la subjetividad. La apuesta de Freire parte del materialismo dialéctico, desarrollado por Marx, en el que en la lógica objeto-sujeto ninguno se superpone al otro y hay una interrelación entre ambos. Freire también se deja acompañar por las ideas latinoamericanas de diversidad y la igualdad social como referente para cualquier propuesta contextualizada en nuestro subcontinente, ello lo asume a partir del pensamiento latinoamericano de la liberación que se manifestaba en su época. (Rivas, 2018, p. 71)

Es así como, para Freire, la escuela debe ser un espacio donde se construya y se pueda vivir la igualdad y se dé el crecimiento de los desfavorecidos y marginados por el sistema dominante. Para él, es de suma importancia la dialéctica; es decir, en los procesos educativos, es necesaria la relación entre el sujeto y objeto, entre la conciencia y naturaleza, y entre la teoría y la práctica (Carbonell, 2015), y la consideración de las contradicciones que surgen en el devenir histórico de la sociedad. La propuesta de Freire nace en su ejercicio como docente y, por consiguiente, tiene clara la necesidad de teorizar desde su práctica profesional, y de practicar sus conclusiones teóricas en los espacios donde nacieron.

Es precisamente en su práctica docente, donde desarrolla su famosa Pedagogía del Oprimido, la cual, según Freire es aquella que debe ser elaborada con el oprimido y no para él (Freire, 2005). La intención de esta pedagogía es que exista una importante participación 
de las personas en su proceso educativo, y que este pueda beneficiarles para su liberación y, a la vez, para su transformación como sujetos activos. Sin embargo, existe una educación bancaria, que busca convertir a las personas en recipientes que deben ser "llenados" por el educador (Freire, 2005). En este tipo de educación, el papel del estudiante es la del oprimido, que no tiene otra opción que ser "educado" dócil y pacientemente por una persona que, según lo bancario, conoce y sabe lo que enseña. Por tal motivo, la pedagogía del oprimido surge como una alternativa ante la educación bancaria.

Para la pedagogía liberadora de Freire, la tarea docente no está relacionada con la simple transmisión de contenidos, sino que debe ir hacia el empoderamiento de los estudiantes, con la intención de que ellos puedan convertirse en actores principales del desarrollo social, político, económico, cultural de sus pueblos. Se busca entonces, que el alumnado pueda, desde sus contextos específicos, luchar contra la opresión, la marginación, la injusticia y todos los males del sistema dominante, y por esta razón esta clase de educación puede llamarse, una educación liberadora (Carbonell, 2015).

De esta manera, la pedagogía propuesta por Freire plantea, al igual que las epistemologías del sur,

La búsqueda de conocimientos y de criterios de validez del conocimiento que otorguen visibilidad y credibilidad a las prácticas cognitivas de las clases, de los pueblos y de los grupos sociales que han sido históricamente victimizados, explotados y oprimidos, por el colonialismo y el capitalismo globales. (Santos, 2009, p. 12)

En este sentido, la educación liberadora debe partir del diálogo entre los miembros de la comunidad educativa. Para Freire (2005), "el diálogo crítico y liberador, dado que supone la acción, debe llevarse a cabo con los oprimidos, cualquiera sea el grado en que se encuentra la lucha por su liberación" (p. 67). Los estudiantes entonces, en este tipo de pedagogía, tienen una voz y se les motiva a utilizarla. No son oyentes más, que como en la educación bancaria, solo escuchan la "sabiduría" de otro, sino que ahora se les invita a participar, en forma activa, del diálogo. Por consiguiente, "la educación así entendida es un proceso interrelacional, dialéctico y dialógico en contra del sujeto universal, obediente y liberal" (Gómez, 2018, p. 107). 
En términos generales, Freire nos invita a reflexionar sobre nuestro quehacer profesional en los espacios de enseñanza y aprendizaje, pero no dejándolo en el mero análisis, sino que debe ser llevado a la práctica, con el fin de generar cambios y transformaciones, realmente pertinentes y significativas para los diversos miembros de las comunidades educativas. Uno de los mayores ejercicios que debe llevarse a la práctica en los espacios educativos es la concientización, la cual permite que "las personas, a partir de su experiencia cotidiana compartida, adquieren una conciencia crítica de sí mismas y de la realidad, que transforman en acción" (Carbonell, 2015, p. 66).

En este sentido, las aulas son espacios oportunos para abrir mesas de diálogo donde los participantes de la construcción del aprendizaje puedan exponer sus experiencias y compararlas con las de los otros. Este tipo de ejercicio permitirá encontrar líneas de análisis en común y, a la vez, generará la tan importante y necesaria concientización, la cual favorece el andamiaje de propuestas que se desarrollan en el colectivo $\mathrm{y}$, que, al mismo tiempo, son llevadas a la práctica, con la intención de lograr cambios importantes en las diversas realidades de opresión y marginación de grupos sociales.

\section{Aportes a la educación superior costarricense}

Las pedagogías críticas muestran un interés por entender a la institución educativa como un espacio de la comunidad y para ella, donde todos los actores puedan participar y crear espacios para la liberación de los marginados. Además, buscan el avance hacia una sociedad cada vez más justa, equitativa y democrática. Este tipo de pedagogías tienen la idea de comprender las diversas culturas, para acercarse de manera adecuada a cada una y, en cada una de ellas, poder aportar para lograr espacios de mayor libertad en las áreas que el ser humano necesita para vivir mejor (Carbonell, 2015).

En el caso de la pedagogía crítica propuesta por Paulo Freire, podemos encontrar una gran lista de aportes, que esta clase de pedagogía puede brindar para la educación superior costarricense en la actualidad. Algunos autores han tratado de expresar herencias que el pensamiento freireano ha ofrecido a la educación, tales como "la dialogicidad en acción, la pedagogía de la pregunta, el binomio reflexión-acción y la alfabetización crítica" (Olivares, 2020, p. 66). Este tipo de aportes son 
necesarios e importantes en el quehacer pedagógico universitario. No obstante, en este ensayo se profundizará en las siguientes tres contribuciones específicas a la educación universitaria costarricense: el diálogo, el pensamiento dialéctico y la transformación.

En primer lugar, Freire desarrolla de manera amplia la necesidad del diálogo en medio de una pedagogía que busque la liberación del oprimido. En este tipo de pedagogía, tanto el docente como el estudiante tienen palabra en el proceso de enseñanza y aprendizaje. No es un espacio vertical, donde el docente habla sin control, como en la educación bancaria, sino que se convierte en una esfera de diálogo horizontal, donde todas y todos los individuos pueden y deben aportar desde sus propias experiencias, realidades y contextos. Para esto, Freire explica que lo primero que debe suceder, es que el docente aprenda a escuchar (Olivares, 2020).

En medio de este proceso de diálogo, "no hay docencia sin discencia; lo cual significa que en el espacio de aprendizaje ninguno de los sujetos toma la posición de objeto" (Valdivia, 2019, p. 41), pues "quien enseña aprende al enseñar y quien aprende enseña al aprender" (Freire, 2004, según se cita en Valdivia, 2019, p. 41). De esta manera, ambos sujetos se encuentran en la misma posición y nutren el diálogo desde sus diferentes realidades. Esto favorece el alejamiento de la mera transmisión de contenidos y el aprovechamiento de la comunicación en los espacios educativos (Gómez, 2018).

En Costa Rica, es urgente una educación basada en el diálogo, donde todos los miembros de la comunidad educativa puedan hablar desde sus contextos y realidades específicas. Es importante que en las aulas universitarias se formen personas que puedan hablar críticamente, pero también que puedan escuchar. Si lo que se pretende es la transformación social, es necesario que todos tengan voz, pero que también tengan oído, para que, de esta manera, de verdad se permita el diálogo en todos los diferentes espacios sociales. Si desde las aulas universitarias se promueve un diálogo genuino, se estará contribuyendo, de manera significativa, al cambio de una sociedad cuya base sea el respeto, la inclusión y la democracia.

Por otra parte, cuando se analiza el pensar dialéctico de Freire, se puede entender la necesidad que existe en la constante reflexión sobre lo que se piensa, se habla y se hace en la educación. Para Freire, la reflexión debe ir acompañada siempre de la acción. No es correcto 
que la reflexión se quede en un pensamiento y mucho menos en meras palabras, pues tal situación se vuelve en una farsa (Freire, 2005). La reflexión se puede compartir en palabras, pero debe llevar a la acción concreta en beneficio de las clases oprimidas, específicamente debe resultar en un auténtico compromiso a favor de la lucha por la liberación (Freire, 2005).

Los espacios educativos son para que tanto el docente como el estudiante se identifiquen en la lucha por su liberación. Para esto, es indispensable que ambos piensen y reflexionen en torno a sus experiencias y realidades concretas. Es aquí donde cobra relevancia enseñar a preguntar, lo cual pretende que el estudiante no asuma una posición conformista de recibir respuesta a todo, sino que más bien desde él mismo pueda reflexionar sobre las preguntas que puedan surgir en el camino de aprendizaje. Según Olivares (2020), "enseñar a preguntar equivale a enseñar a pensar" (p. 68).

De acuerdo con lo anterior, las universidades costarricenses deben tomar en cuenta la reflexión acompañada de acción. En un primer momento, el docente debe convertirse en un sujeto reflexivo de su quehacer educativo, entendiendo que es "un ser histórico e inacabado que siempre puede mejorar" (Valdivia, 2019, p. 41). Además, los mismos estudiantes tienen que comprender que la educación superior no es justo para obtener un título más, sino un espacio para crecer integralmente en libertad y en búsqueda de la transformación. En general, la institución universitaria debe convertirse en un ámbito de constante reflexión, que siempre pueda ir de la mano con la acción. Esto es esencial para el buen desarrollo de la educación superior costarricense.

Por esta razón, cada vez más las casas de estudios superiores tienen que relacionarse mayormente con la realidad del país, y, sobre todo, de los grupos sociales marginados e invisibilizados. Tanto docentes como estudiantes tienen la tarea y responsabilidad de reflexionar acerca de los problemas que se viven en la actualidad, pero no solo dejándolo como un análisis más, sino procurando diseñar líneas de acción concretas y adecuadas para cada uno de los sectores afectados. La universidad tiene el llamado a incidir de forma positiva en la sociedad, a la cual se debe. Es una oportunidad para las universidades costarricenses de demostrar lo importante de su existencia y el impacto que ellas tienen en el bienestar del país. 
En tercer lugar, Freire aporta un tema esencial en su pensamiento pedagógico, la transformación. Es necesario recordar, que este es un punto en común dentro de las pedagogías críticas. Sin embargo, existe un énfasis importante en el desarrollo del pensamiento freireano. Dentro de la pedagogía crítica de Freire (2005), "los sujetos se encuentran, para la transformación del mundo, en colaboración" (p. 217). Aquí juega un papel relevante el diálogo, el cual permite que los sujetos de forma horizontal presenten sus pensamientos, experiencias y realidad cotidiana. En ese sentido, para Freire (2005),

La pedagogía del oprimido, como pedagogía humanista y liberadora, tendrá, pues, dos momentos distintos aunque interrelacionados. El primero, en el cual los oprimidos van descubriendo el mundo de la opresión y se van comprometiendo, en la praxis, con su transformación y, el segundo, en que una vez transformada la realidad opresora, esta pedagogía deja de ser del oprimido y pasa a ser la pedagogía de los hombres en proceso de permanente liberación. (p. 55)

Por consiguiente, el profesorado universitario tiene que recordar su papel primordial en la transformación de los sujetos, pero no solo de los estudiantes, sino también en la transformación social (Zúñiga, 2011). Por tal motivo, las aulas universitarias deben ser espacios para formar y transformar personas críticas, reflexivas, las cuales estén comprometidas con el cambio que las diferentes esferas sociales requieren. Sin lugar a duda, para formar personas como estas es indispensable un sistema político-legal que permita y promueva espacios de crítica y reflexión. Si la institución universitaria pierde su autonomía, caerá en el peligro de tener que reproducir pensamientos dominantes impuestos por la clase que mantiene el poder.

Por tal motivo, es importante que las universidades costarricenses involucren cada vez más a los diversos integrantes de la comunidad educativa en la transformación interna de toda institución. Cada momento es el adecuado para que los participantes de los procesos universitarios comprendan la importancia que tiene la autonomía de las casas de estudios superiores y los grandes peligros de perderla. Parte de la transformación social que debe ser generada desde las universidades es la correcta comprensión sobre la autonomía universitaria; existen 
detractores que enseñan asuntos falsos y erróneos sobre ella y hacen caer a la población en un desconocimiento. Es por ello, que la tarea de la universidad en la actualidad es procurar la transformación social en torno al tema de la autonomía universitaria, buscando cada día el apoyo de la mayoría de la población.

\section{A modo de conclusión}

Como se ha explicado hasta el momento, las ideas pedagógicas propuestas por Paulo Freire son pertinentes para la aplicación en la educación superior costarricense de la actualidad. Dentro de sus grandes aportes a la pedagogía en general, en este ensayo se han rescatado tres de ellos: el diálogo, el pensar dialéctico y la transformación. Estos aspectos son constantemente enfatizados en el pensamiento freireano, por tal razón es necesario hablar de ellos y, al mismo tiempo, analizar su pertinencia para el contexto nacional. Tanto el diálogo, el pensar dialéctico y la transformación son necesarios y urgentes en la universidad pública de Costa Rica, ante los grandes desafíos que se presentan en día.

En este sentido, cuando se enseña y aprende a dialogar, las personas comprenden que el derecho de la palabra no solamente está enmarcado para las clases dominantes, sino que todos los sujetos tienen voz, y, por tanto, la capacidad de proponer, argumentar y sostener sus diversas opiniones sobre un tema. Además, el pensamiento dialógico enriquece el análisis y la reflexión por medio de la acción. Es importante, que las personas puedan desarrollar la habilidad de reflexionar sobre los grandes problemas que aquejan a la sociedad; sin embargo, es aún más importante que la acompañen de líneas de acción concretas en busca de cambios reales y pertinentes, pues de nada sirve reflexiones amplias y llenas de argumentación, si nunca se busca la transformación social.

Es así como, los docentes deben jugar un papel de gran relevancia en el proceso de enseñanza y aprendizaje, pues su tarea no se debe enmarcar en la sencilla transmisión del conocimiento, sino que la práctica docente tiene que desarrollar actividades de clase donde estén presentes la crítica, el cuestionamiento, la reflexión, los cuales inviten a los participantes al ejercicio de la liberación y emancipación (Carbonell, 2015). El profesor que desee llevar a sus estudiantes a la crítica reflexiva debe abrazar esta actividad desde su misma práctica profesional. No es posible que el docente invite al estudiantado a reflexionar y 
criticar sobre diversos temas, cuando su propio ejercicio docente sigue sin ningún tipo de transformación. En este sentido, el profesor debe comprometerse con la reflexión, la crítica y la transformación, iniciando por su tarea como profesional en la educación.

Por último, la educación superior debe ser un espacio donde todas y todos encuentren un lugar para crecer y desarrollarse como sujetos libres. Cada ambiente educativo se convierte en un espacio de expresión donde los estudiantes, sin importar su clase social, económica, religiosa o de género, interactúan desde el lugar en donde se ubican dentro de la sociedad, sin que estas diferencias puedan materializarse en desigualdades o jerarquizaciones. En medio de la diversidad presente en la universidad, se permite el desarrollo de espacios más ricos y significativos para todos los participantes. Las pedagogías críticas buscan esta clase de espacios, que permitan a los estudiantes aprender de los otros y encontrar, en medio de la diversidad, un sitio para la libertad del ser humano (Carbonell, 2015). No obstante, debemos preguntarnos si estos espacios se podrán desarrollar en un país donde cada vez más se atenta contra la autonomía y el presupuesto de aquellas instituciones que históricamente han sido promotoras del cambio y la transformación de los grupos vulnerables y excluidos de la sociedad.

\section{Referencias}

Carbonell, J.(2015). Pedagogías del siglo XXI. Alternativas para la innovación educativa. Editorial Octaedro. http://investigacionpedagogicaunam. weebly.com/uploads/1/0/8/8/10888154/pedagog\%C3\%8Cas_del_ siglo_xxi_alternativas_para_la_innovaci\%C $3 \% \mathrm{~B} 2 \mathrm{n}$ _educativa___jaume_carbonell_sebarroja___2015_.pdf

Córdoba, J. (08 de julio de 2020). La mejor forma de extorsionar a una universidad es cercenándole su presupuesto. Semanario Universidad. https://semanariouniversidad.com/universitarias/ la-mejor-forma-de-extorsionar-a-una-universidad-es-cercenandole-su-presupuesto/

Freire, P. (2005). Pedagogía del Oprimido. Siglo XXI Editores.

Gómez, J. (2018). Educación, comunidad y liberación. Comentarios a partir del pensamiento pedagógico de Paulo Freire y Alejandro Cerletti: aportes a la enseñanza de la filosofía. Revista Ensayos Pedagógicos, Edición Especial: Indisciplinando la 
Didáctica, 103-113. https://www.revistas.una.ac.cr/index.php/ ensayospedagogicos/article/view/10851

Olivares, C. (2020). Integración del pensamiento freireano al quehacer universitario: hacia una transformación de la propia praxis. Revista Ensayos Pedagógicos, 15(1), 61-79. https://doi.org/10.15359/ rep.15-1.3

Rivas, O. (2018). Freire y la Pedagogía del Oprimido. Revista Ensayos Pedagógicos, Edición Especial: Indisciplinando la Didáctica, 69-79. https://www.revistas.una.ac.cr/index.php/ ensayospedagogicos/article/view/10849

Santos, B. (2009). Una epistemología del sur. Siglo XXI Editores.

Torres, J. (2017). Políticas educativas y construcción de personalidades neoliberales y neocolonialistas. Ediciones Morata.

Valdivia, S. (2019). Desafíos de la docencia universitaria desde la pedagogía de la autonomía. Revista Ensayos Pedagógicos, 14(2), 29-43. https://doi.org/10.15359/rep.14-2.2

Walsh, C. (2017). Entretejiendo lo pedagógico y lo decolonial: luchas, caminos y siembras de reflexión-acción para resistir, (re)existir $y$ (re)vivir. Editorial Alternativas. https://alternativas.osu.edu/es/ ebooks/catalog/new-ebook.html

Zúñiga, A. (2011). Análisis crítico de la Educación Costarricense a la luz del plan liberador propuesto por Pablo Freire. Revista Ensayos Pedagógicos, 6(2), 43-54. https://www.revistas.una.ac.cr/ index.php/ensayospedagogicos/article/view/5818 